\title{
Fault-Tolerant Resolvability in Some Classes of Subdivision Graphs
}

\author{
Muhammad Faheem, ${ }^{1}$ Zohaib Zahid $\left(\mathbb{D},{ }^{1}\right.$ Dalal Alrowaili ${ }^{D},{ }^{2}$ Imran Siddique $\left(\mathbb{D},{ }^{1}\right.$ \\ and Aiyared Iampan $\mathbb{D}^{3}$ \\ ${ }^{1}$ Department of Mathematics, University of Management and Technology, Lahore 54770, Pakistan \\ ${ }^{2}$ Department of Mathematics, College of Science, Jouf University, P.O. Box: 2014, Sakaka, Saudi Arabia \\ ${ }^{3}$ Department of Mathematics, School of Science, University of Phayao, Mae Ka, Mueang, Phayao 56000, Thailand
}

Correspondence should be addressed to Imran Siddique; imransmsrazi@gmail.com

Received 9 November 2021; Accepted 13 January 2022; Published 18 February 2022

Academic Editor: Gul Rahmat

Copyright (c) 2022 Muhammad Faheem et al. This is an open access article distributed under the Creative Commons Attribution License, which permits unrestricted use, distribution, and reproduction in any medium, provided the original work is properly cited.

\begin{abstract}
The concept of resolving sets (RSs) and metric dimension (MD) invariants have a wide range of applications in robot navigation, computer networks, and chemical structure. RS has been used as a sensor in an indoor positioning system to find an interrupter. Many terminologies in machine learning have also been used to diagnose the interrupter in the systems of marine and gas turbines using sensory data. We proposed a fault-tolerant self-stable system that allows for the detection of an interrupter even if one of the sensors in the chain fails. If the elimination of any element from a RS is still a RS, then the RS is considered as a fault-tolerant resolving set (FTRS), and the fault-tolerant metric dimension (FTMD) is its minimum cardinality. In this paper, we calculated the FTMD of the subdivision graphs of the necklace and prism graphs. We also found that this invariant has constant values for both graphs.
\end{abstract}

\section{Introduction and Preliminaries}

Let $G$ be a connected, undirected, finite, and simple graph with vertex set $V(G)$ and edge set $E(G)$. The number of edges in the shortest $x-y$ path between two vertices $x, y \in V(G)$ is known as the distance $d_{G}(x, y)$ between them. The cardinality of edges that are incident to a vertex $x$ is called degree $d_{G}(x)$ of $x$. Let $W=\left\{w_{1}, w_{2}, \ldots, w_{s}\right\}$ be an ordered subset of $V(G)$ and $y \in V(G)$; then, the $s$-tuple $\left(d_{G}\left(y, w_{1}\right), d_{G}\left(y, w_{2}\right), \ldots, d_{G}\left(y, w_{s}\right)\right)$ is the distance-vector representation of $y$ with respect to $W$ and is denoted by $r(y \mid W)$. If distance-vector representations $r(y \mid W)$ are distinct for every vertex $y$ of $G$, then $W$ is known as the RS. The metric basis of $G$ is the minimum number of elements in a RS, and the cardinality of the basis is called the MD of $G$, denoted by $\beta(G)$. For $W=\left\{w_{1}, w_{2}, \ldots, w_{s}\right\} \subset V(G)$, the $i$ th component of $r(y \mid W)$ is 0 if and only if $y=w_{i}$. So, $W$ would be a RS if $r(x \mid W) \neq r(y \mid W)$ for each pair $x \neq y \in V(G) \backslash W$. The absolute difference representation consists of $s$-vector
$\left(\left|d_{G}\left(x, w_{1}\right)-d_{G}\left(y, w_{1}\right)\right|, \ldots,\left|d_{G}\left(x, w_{s}\right)-d_{G}\left(y, w_{s}\right)\right|\right)$ for any $x, y \in V(G)$ with respect to $W$, and it is denoted by $A D((x, y) \mid W)$. The absolute difference representation is another concept to define the RS. If $A D((x, y) \mid W)$ has at least one nonzero element in its $s$-vector for every $x \neq y \in V(G)$, then $W$ is called the RS. For more details, we refer to $[1,2]$.

In 1975, when Slater was working with Coast Guard Loran and US Sonar (long range aids to navigation) station, he described the notion of RSs in graphs $[3,4]$. Harary and Melter independently described this idea in 1976 [5]. The RSs were introduced to locate the interrupter in a computer network. After that, in 2003, Chartrand and Zhang used metric bases in the fields of biology, robotics, and chemistry $[6,7]$. Many techniques have been used in machine learning to find the interrupter using sensory data. In 2021, Tan et al. compared the data-driven and model-driven methods for the diagnosis of interrupters in marine machinery systems [8]. 
It will be a difficult task to locate an interrupter if one of the sensors does not work properly. To tackle such problems, Hernando et al. gave the idea of a FTRS [9]. If the elimination of any element from a RS is again a RS, then the RS is considered as a FTRS. Formally, for any RS $W^{\prime}$ of $G$, if $W^{\prime}\{w\}$ is also a RS for any $w \in W^{\prime}$, then $W^{\prime}$ is called a FTRS of $G$. In other words, $A D\left((x, y) \mid W^{\prime}\right)$ contains at least two elements that are nonzero in the $s$-vector for all $x \neq y \in V(G)$. The FTMD is the minimum cardinality of a FTRS, and it is denoted by $\beta^{\prime}(G)$.

Hernando et al. in [9] studied the concept of the FTMD and computed it for the tree graph. They also found an important upper bound for the FTMD of graph $G$, which is $\beta^{\prime}(G) \leq \beta(G)\left(1+2.5^{\beta(G)-1}\right)$. Voronov in [10] determined the upper bound for the FTMD of the king's graph. Hussain et al. in [11] proved that the FTMD is constant for the antiweb graph and unbounded for the gear and antiweb gear graphs.

Raza et al. in [12] studied the bounds for the FTMD of some families of convex polytopes. In [13], Raza et al. proved some bounds for the FTMD of extended Petersen, antiprism, and squared cycle graphs. Raza et al. in [14] found some bounds for the FTMD by considering the graphs of $(m, n)$-dimension grid networks, hexagonal networks, and honeycomb networks. Zheng et al. in [15] calculated the precise values for the FTMD of the generalized wheels and some families of convex polytopes. Afzal and others in [16] gave some important results and proved that there exist some families of convex polytopes, which have unbounded MD and FTMD. Basak et al. in [17] calculated the FTMD for the circulant graph. Somasundari and Raj in [18] calculated the fault-tolerant resolvability for the interconnections of oxide networks.

Javaid et al. in [19] computed the exact value of the FTMD of the cycle graph. Hayat et al. in [20] calculated some upper bounds for the FTMD for $r$-dimensional bens, $r$-dimensional butterfly, and silicate networks. Simic et al. in [21] contributed great work about the FTMD and computed the precise value for the grid graph. Laxman in [22] computed the lower bound of the FTMD for the cube of the path graph. Recently, the FTMD for the line graphs was studied by Guo et al. in [23], and they computed it for the line graphs of the prism and necklace graphs.

The subdivision graph $S(G)$ of any graph $G$ can be obtained by adding a new vertex to each edge of the graph $G$ as shown in Figures 1 and 2. In this paper, we computed the FTMD of the subdivision graphs of the necklace and the prism graphs. The results of the FTMD in subdivision graphs are known only for cycle and path graphs as shown in the following theorem.

Theorem 1. For any $n \geq 2$ and $n \geq 3$, we have $\beta^{\prime}\left(S\left(P_{n}\right)\right)=2$ and $\beta^{\prime}\left(S\left(C_{n}\right)\right)=3$, respectively, where $P_{n}$ is the path graph and $C_{n}$ is the cycle graph.

Since it is not an easy task to calculate $\beta^{\prime}(G)$ for any graph $G$, Estrado-Moreno et al. computed some important bounds on $\beta^{\prime}(G)$ of every graph $G$ as follows.

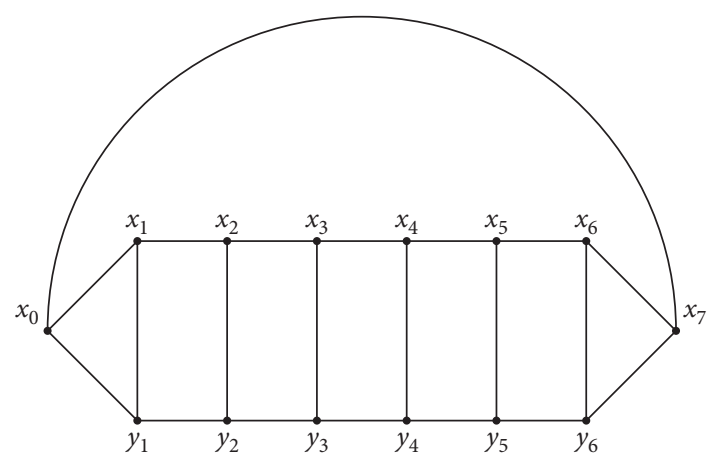

FIgURE 1: Subdivision of necklace graph $S\left(N_{e_{6}}\right)$.

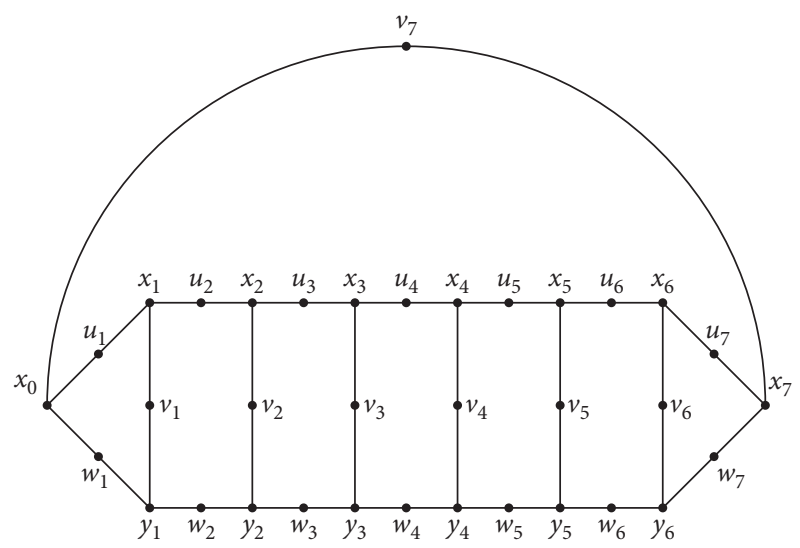

Figure 2: Subdivision of prism graph $S\left(Y_{n}\right)$.

Lemma 1 (see [24]). Let $G$ be any graph; then, we have $\beta(G)<\beta^{\prime}(G)$.

Lemma 2 (see [24]). Let $G \neq P_{n}$ be any graph; then, we have $\beta^{\prime}(G) \geq 3$.

Khuller et al. [25] gave an essential result for every graph $G$ with 2 of its MD as follows.

Lemma 3 (see [25]). Let $\beta(G)=2$, for any graph $G$, and let $\left\{v_{1}, v_{2}\right\} \subset V(G)$ be a RS in G. Then, $d_{G}\left(v_{1}\right) \leq 3$ and $d_{G}\left(v_{2}\right) \leq 3$.

Therefore, similar property works for every graph $G$ with 3 of its $\beta^{\prime}(G)$ as presented in the following lemma.

Lemma 4. Let $\beta^{\prime}(G)=3$, for any graph $G$, and let $\left\{v_{1}, v_{2}, v_{3}\right\} \subset V(G)$ be a FTRS in G. Then, $d_{G}\left(v_{1}\right) \leq 3$, $d_{G}\left(v_{2}\right) \leq 3$, and $d_{G}\left(v_{3}\right) \leq 3$.

We will discuss and compute the precise results of $\beta\left(S\left(N_{e_{n}}\right)\right)$ as well as $\beta^{\prime}\left(S\left(N_{e_{n}}\right)\right)$, where $N_{e_{n}}$ is a necklace graph in Section 2. We will calculate the exact value of $\beta^{\prime}\left(S\left(Y_{n}\right)\right)$ by considering $Y_{n}$ as a prism graph in Section 3. 


\section{The Fault-Tolerant Metric Dimension for the Subdivision of the Necklace Graph}

The necklace graph $N_{e_{n}}$ for $n \geq 3$ consists of the vertices $\left\{x_{i}, y_{j}: 0 \leq i \leq n+1,1 \leq j \leq n\right\}$ as shown in Figure 3.

Now, to compute $\beta^{\prime}\left(S\left(N_{e_{n}}\right)\right)$, we need to convert the necklace graph into its subdivision graph. The subdivision graph of the necklace graph $S\left(N_{e_{n}}\right)$ is shown in Figure 1.

The result about the MD for the subdivision graph of the necklace graph is presented in the following theorem.

Theorem 2. For any integer $n \geq 3$, we have $\beta\left(S\left(N_{e_{n}}\right)\right)=3$.
Proof. Following are the cases to compute the required results:

Case 1 (when $n$ is odd).

Take $W=\left\{x_{0}, u_{n+1 / 2}, v_{n+3 / 2}\right\} \subseteq V\left(S\left(N_{e_{n}}\right)\right)$ for all odd integers $n \geq 3$. We need to prove that $W$ is the RS for the graph $S\left(N_{e_{n}}\right)$. To prove this, we give distance-vector representations of every vertex in $S\left(N_{e_{n}}\right)$. Representation of the vertices $x_{\rho}$ where $0 \leq \rho \leq n+1$ is

$$
r\left(x_{\rho} \mid W\right)= \begin{cases}(0, n, 2+n), & \text { if } \rho=0, \\ (2 \rho,-2 \rho+n, 4-2 \rho+n), & \text { if } 1 \leq \rho \leq \frac{n-1}{2}, \\ (1+n, 1,3), & \text { if } \rho=\frac{1+n}{2}, \\ \left(2(-\rho+2+n), 2\left(\rho-\frac{n}{2}\right), 2\left(\frac{-n}{2}-1+\rho\right)\right), & \text { if } \frac{3+n}{2} \leq \rho \leq 1+n .\end{cases}
$$

Representation of the vertices $u_{\rho}$ where $1 \leq \rho \leq 1+n$ is

$$
r\left(u_{\rho} \mid W\right)= \begin{cases}(-1+2 \rho,-2 \rho+1+n,-2 \rho+5+n), & \text { if } 1 \leq \rho \leq \frac{1+n}{2}, \\ (2+n, 2,2), & \text { if } \rho=\frac{3+n}{2}, \\ (5+2(-\rho+n),-n-1+2 \rho,-n-3+2 \rho), & \text { if } \frac{5+n}{2} \leq \rho \leq 1+n .\end{cases}
$$

Representation of the vertices $w_{\rho}$ where $1 \leq \rho \leq 1+n$ is

$$
r\left(w_{\rho} \mid W\right)= \begin{cases}(-1+2 \rho,-2 \rho+3+n,-2 \rho+5+n), & \text { if } 1 \leq \rho \leq \frac{n-1}{2}, \\ (n, 4,4), & \text { if } \rho=\frac{1+n}{2}, \\ (2+n, 4,2), & \text { if } \rho=\frac{3+n}{2}, \\ (5-2 \rho+2 n,-n+1+2 \rho,-3-n+2 \rho), & \text { if } \frac{5+n}{2} \leq \rho \leq 1+n .\end{cases}
$$




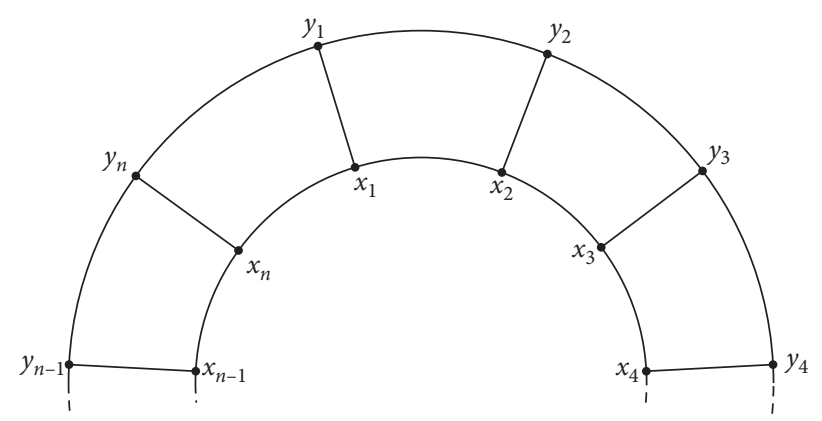

Figure 3: Necklace graph $N_{e_{6}}$.

Representation of the vertices $v_{\rho}$ where $1 \leq \rho \leq 1+n$ is

$$
r\left(v_{\rho} \mid W\right)= \begin{cases}(1+2 \rho,-2 \rho+1+n,-2 \rho+5+n), & \text { if } 1 \leq \rho \leq \frac{n-1}{2}, \\ (2+n, 2,4), & \text { if } \rho=\frac{1+n}{2}, \\ (2+n, 4,0), & \text { if } \rho=\frac{3+n}{2}, \\ (-2 \rho+5+2 n,-n+1+2 \rho,-n-1+2 \rho), & \text { if } \frac{5+n}{2} \leq \rho \leq n, \\ (1, n+1,1+n), & \text { if } \rho=1+n .\end{cases}
$$

Representation of the vertices $y_{\rho}$ where $1 \leq \rho \leq n$ is

$$
r\left(y_{\rho} \mid W\right)= \begin{cases}(2 \rho,-2 \rho+2+n, 4-2 \rho+n), & \text { if } 1 \leq \rho \leq \frac{n-1}{2}, \\ (1+n, 3,3), & \text { if } \rho=\frac{1+n}{2}, \\ (-2 \rho+4+2 n,-n+2+2 \rho,-n-2+2 \rho), & \text { if } \frac{3+n}{2} \leq \rho \leq n .\end{cases}
$$

The distance-vector representations are distinct for all the vertices of $S\left(N_{e_{n}}\right)$. So, $W$ is the RS. Hence, $\beta\left(S\left(N_{e_{n}}\right)\right) \leq 3$. Now, in order to prove that $\beta\left(S\left(N_{e_{n}}\right)\right) \geq 3$, suppose contrary that $\beta\left(S\left(N_{e_{n}}\right)\right)=2$, and according to Lemma 3, we have the following possibilities:

(1) Let $W=\left\{x_{i}, x_{j}\right\} \subset V\left(S\left(N_{e_{n}}\right)\right)$ for $0 \leq i<j \leq n+1 / 2$; then, we have the following subcases:

(i) $A D\left(\left(u_{n+1}, w_{n+1}\right) \mid W\right)=(0,0)$ for $j \neq n+1 / 2$.

(ii) $A D\left(\left(u_{n+3 / 2}, v_{n+1 / 2}\right) \mid W\right)=(0,0)$ for $j=n+1 / 2$. So, $W$ is not the RS.
(2) Let $W=\left\{u_{i}, u_{j}\right\} \subset V\left(S\left(N_{e_{n}}\right)\right)$ for $0 \leq i<j \leq$ $n+1 / 2$; then, we have the following subcases:

(i) $A D\left(\left(u_{n+1}, w_{n+1}\right) \mid W\right)=(0,0)$ for $j \neq n+1 / 2$.

(ii) $A D\left(\left(u_{n+3 / 2}, v_{n+1 / 2}\right) \mid W\right)=(0,0)$ for $j=n+1 / 2$. So, $W$ is not the RS.

(3) Let $W=\left\{x_{i}, x_{j}\right\} \subset V\left(S\left(N_{e_{n}}\right)\right)$ for $0 \leq i \leq n+1 / 2$ and $n+3 / 2 \leq j \leq n+1$; then, we have the following subcases:

(a) When $n=3$ :

(i) $A D\left(\left(v_{3}, w_{3}\right) \mid W\right)=(0,0)$ for $j \neq 3$. 
(ii) $A D\left(\left(u_{3}, v_{3}\right) \mid W\right)=(0,0)$ for $i=0$ and $j=3$.

(iii) $A D\left(\left(u_{4}, v_{3}\right) \mid W\right)=(0,0)$ for $i=1,2$ and $j=3$.

(b) When $n \geq 5$ :

(i) $A D\left(\left(v_{n}, w_{n}\right) \mid W\right)=(0,0)$ for $j \neq n$.

(ii) $A D\left(\left(u_{n}, v_{n}\right) \mid W\right)=(0,0)$ for $0 \leq i \leq n-3 / 2$ and $j=n$.

(iii) $A D\left(\left(v_{1}, w_{2}\right) \mid W\right)=(0,0)$ for $n-1 / 2 \leq i \leq$ $n+1 / 2$ and $j=n$.

So, $W$ is not the RS.

(4) Let $W=\left\{u_{i}, u_{j}\right\} \subset V\left(S\left(N_{e_{n}}\right)\right)$ for $1 \leq i \leq n+1 / 2$ and $n+3 / 2 \leq j \leq n+1$; then, we have the following subcases:

(a) When $n=3$ :

(i) $A D\left(\left(v_{1}, v_{4}\right) \mid W\right)=(0,0)$ for $i=1$ and $j=3$.

(ii) $A D\left(\left(u_{3}, w_{4}\right) \mid W\right)=(0,0)$ for $i=1$ and $j=4$.

(iii) $A D\left(\left(w_{2}, w_{3}\right) \mid W\right)=(0,0)$ for $i=2$ and $j=3$.

(iv) $A D\left(\left(u_{1}, v_{2}\right) \mid W\right)=(0,0)$ for $i=2$ and $j=4$.

(b) When $n=5$ :

(i) $A D\left(\left(v_{5}, w_{5}\right) \mid W\right)=(0,0)$ for $j \neq 5,6$.

(ii) $A D\left(\left(x_{3}, y_{5}\right) \mid W\right)=(0,0)$ for $i=1$ and $j=5$.

(iii) $A D\left(\left(v_{3}, v_{6}\right) \mid W\right)=(0,0)$ for $i=2$ and $j=5$.

(iv) $A D\left(\left(v_{1}, w_{2}\right) \mid W\right)=(0,0)$ for $i=3$ and $j=5$.

(v) $A D\left(\left(u_{5}, v_{5}\right) \mid W\right)=(0,0)$ for $i=1$ and $j=6$.

(vi) $A D\left(\left(v_{1}, u_{3}\right) \mid W\right)=(0,0)$ for $i=2$ and $j=6$.

(vii) $A D\left(\left(v_{1}, w_{2}\right) \mid W\right)=(0,0)$ for $i=3$ and $j=6$.

(c) When $n \geq 7$ :

(i) $A D\left(\left(v_{n}, w_{n}\right) \mid W\right)=(0,0)$ for $j \neq n, n+1$.

(ii) $A D\left(\left(u_{n-1}, v_{n-1}\right) \mid W\right)=(0,0)$ for $i=1,2$ and $j=n$.

(iii) $A D\left(\left(v_{1}, w_{2}\right) \mid W\right)=(0,0)$ for $3 \leq i \leq(n+1) / 2$ and $j=n$.

(iv) $A D\left(\left(u_{n}, v_{n}\right) \mid W\right)=(0,0)$ for $i=1,2$ and $j=n+1$.

(v) $A D\left(\left(v_{1}, w_{2}\right) \mid W\right)=(0,0)$ for $3 \leq i \leq(n+1) / 2$ and $j=n+1$.

So, $W$ is not the RS.

(5) Let $W=\left\{x_{i}, u_{j}\right\} \subset V\left(S\left(N_{e_{n}}\right)\right)$ for $0 \leq i \leq n+1 / 2$ and $1 \leq j \leq n+1 / 2$; then, $A D\left(\left(v_{n}, w_{n}\right) \mid W\right)=(0,0)$. So, $W$ is not the RS.

(6) Let $W=\left\{x_{i}, v_{j}\right\} \subset V\left(S\left(N_{e_{n}}\right)\right)$ for $0 \leq i \leq n+1 / 2$ and $1 \leq j \leq n+1 / 2$; then, we have the following subcases:

(i) $A D\left(\left(u_{n+1}, w_{n+1}\right) \mid W\right)=(0,0)$ for $i \neq n+1 / 2$.

(ii) $A D\left(\left(u_{n+5 / 2}, v_{n+3 / 2}\right) \mid W\right)=(0,0)$ for $i=n+1 / 2$.

So, $W$ is not the RS.

(7) Let $W=\left\{u_{i}, v_{j}\right\} \subset V\left(S\left(N_{e_{n}}\right)\right)$ for $1 \leq i \leq n+1 / 2$ and $1 \leq j \leq n+1 / 2$; then, we have the following subcases: (i) $A D\left(\left(u_{n+1}, w_{n+1}\right) \mid W\right)=(0,0)$ for $i \neq n+1 / 2$.

(ii) $A D\left(\left(u_{n+5 / 2}, v_{n+3 / 2}\right) \mid W\right)=(0,0)$ for $i=n+1 / 2$. So, $W$ is not the RS.

(8) Let $W=\left\{x_{i}, y_{j}\right\} \subset V\left(S\left(N_{e_{n}}\right)\right)$ for $0 \leq i \leq n+1 / 2$ and $1 \leq j \leq n+1 / 2$; then, we have the following subcases:

(i) $A D\left(\left(u_{n+1}, w_{n+1}\right) \mid W\right)=(0,0)$ for $i \neq n+1 / 2$ and $j \neq n+1 / 2$.

(ii) $A D\left(\left(u_{n+1 / 2}, v_{n+1 / 3}\right) \mid W\right)=(0,0)$ for $i=n+1 / 2$ and $j \neq n+1 / 2$.

(iii) $A D\left(\left(u_{n}, v_{n}\right) \mid W\right)=(0,0) \quad$ for $\quad i=0 \quad$ and $j=n+1 / 2$.

(iv) $A D\left(\left(v_{n+1 / 2}, w_{n+1 / 2}\right) \mid W\right)=(0,0)$ for $1 \leq i \leq$ $n-1 / 2$ and $j=n+1 / 2$.

(v) $A D\left(\left(u_{n+1 / 2}, u_{n+3 / 2}\right) \mid W\right)=(0,0)$ for $i=n+1 / 2$ and $j=n+1 / 2$.

So, $W$ is not the RS.

(9) Let $W=\left\{u_{i}, w_{j}\right\} \subset V\left(S\left(N_{e_{n}}\right)\right)$ for $1 \leq i \leq n+1 / 2$ and $1 \leq j \leq n+1 / 2$; then, we have the following subcases:

(a) When $n=3$ :

(i) $A D\left(\left(u_{4}, w_{4}\right) \mid W\right)=(0,0)$ for $i=1$ and $j=1$.

(ii) $A D\left(\left(u_{1}, v_{1}\right) \mid W\right)=(0,0)$ for $i=2$ and $j=1$.

(iii) $A D\left(\left(v_{1}, w_{1}\right) \mid W\right)=(0,0)$ for $i=1$ and $j=2$.

(iv) $A D\left(\left(x_{1}, x_{2}\right) \mid W\right)=(0,0)$ for $i=2$ and $j=2$.

(10) Let $W=\left\{u_{i}, y_{j}\right\} \subset V\left(S\left(N_{e_{n}}\right)\right)$ for $1 \leq i \leq n+1 / 2$ and $1 \leq j \leq n+1 / 2$; then, we have the following subcases:

(i) $A D\left(\left(u_{n+1}, w_{n+1}\right) \mid W\right)=(0,0)$ for $i \neq n+1 / 2$ and $j \neq n+1 / 2$.

(ii) $A D\left(\left(u_{n-1 / 2}, v_{n-1 / 2}\right) \mid W\right)=(0,0)$ for $i=n+1 / 2$ and $j \neq n-1 / 2, n+1 / 2$.

(iii) $A D\left(\left(u_{n-1 / 2}, v_{n+1 / 2}\right) \mid W\right)=(0,0)$ for $i=n+1 / 2$ and $j=n-1 / 2$.

(iv) $A D\left(\left(v_{n+1 / 2}, w_{n+1 / 2}\right) \mid W\right)=(0,0)$ for $i \neq n+1 / 2$ and $j=n+1 / 2$.

(v) $A D\left(\left(w_{n+1 / 2}, w_{n+3 / 2}\right) \mid W\right)=(0,0)$ for $i=n+1 / 2$ and $j=n+1 / 2$.

So, $W$ is not the RS.

We deduce to contradict in all the possibilities. So, there is no RS with cardinality 2 . This shows that $\beta\left(S\left(N_{e_{n}}\right)\right) \geq 3$. Hence, $\beta\left(S\left(N_{e_{n}}\right)\right)=3$.

Case 2 (when $n$ is even).

Take $W=\left\{x_{0}, u_{n / 2}, y_{n / 2}\right\} \subseteq V\left(S\left(N_{e_{n}}\right)\right)$ for all even integers $n \geq 4$. Representation of the vertices $x_{\rho}$ where $0 \leq \rho \leq 1+n$ is 


$$
r\left(x_{\rho} \mid W\right)= \begin{cases}(0,-1+n, n), & \text { if } \rho=0, \\ (2 \rho,-2 \rho-1+n,-2 \rho+2+n), & \text { if } 1 \leq \rho \leq \frac{n-2}{2}, \\ (n, 1,2), & \text { if } \rho=\frac{n}{2}, \\ (4-2 \rho+2 n,-n+1+2 \rho, 2-n+2 \rho), & \text { if } \frac{2+n}{2} \leq \rho \leq n, \\ (2, n+1,2+n), & \text { if } \rho=1+n .\end{cases}
$$

Representation of the vertices $u_{\rho}$ where $1 \leq \rho \leq 1+n$ is

$$
r\left(u_{\rho} \mid W\right)= \begin{cases}(-1+2 \rho,-2 \rho+n, 3-2 \rho+n), & \text { if } 1 \leq \rho \leq \frac{n}{2}, \\ (1+n, 2,3), & \text { if } \rho=\frac{2+n}{2}, \\ (-2 \rho+5+2 n,-n+2 \rho,-n+1+2 \rho), & \text { if } \frac{4+n}{2} \leq \rho \leq 1+n .\end{cases}
$$

Representation of the vertices $w_{\rho}$ where $1 \leq \rho \leq 1+n$ is

$$
r\left(w_{\rho} \mid W\right)= \begin{cases}(-1+2 \rho,-2 \rho+2+n,-2 \rho+1+n), & \text { if } 1 \leq \rho \leq \frac{n-2}{2} \\ (-1+2 \rho, 4,1), & \text { if } \frac{n}{2} \leq \rho \leq \frac{2+n}{2} \\ (5-2 \rho+2 n,-n+2+2 \rho,-1-n+2 \rho), & \text { if } \frac{4+n}{2} \leq \rho \leq n \\ (3, n+2,1+n), & \text { if } \rho=1+n .\end{cases}
$$

Representation of the vertices $v_{\rho}$ where $1 \leq \rho \leq 1+n$ is

$$
r\left(v_{\rho} \mid W\right)= \begin{cases}(2 \rho+1,-2 \rho+n,-2 \rho+1+n), & \text { if } 1 \leq \rho \leq \frac{n-2}{2}, \\ (n+1,2,1), & \text { if } \rho=\frac{n}{2}, \\ (5-2 \rho+2 n, 2-n+2 \rho,-n+1+2 \rho), & \text { if } \frac{2+n}{2} \leq \rho \leq n, \\ (1, n, n+1), & \text { if } \rho=1+n .\end{cases}
$$


Representation of the vertices $y_{\rho}$ where $1 \leq \rho \leq n$ is

$$
r\left(y_{\rho} \mid W\right)= \begin{cases}(2 \rho,-2 \rho+1+n,-2 \rho+n), & \text { if } 1 \leq \rho \leq \frac{n-2}{2} \\ (n, 3,0), & \text { if } \rho=\frac{n}{2}, \\ (4-2 \rho+2 n,-n+3+2 \rho,-n+2 \rho), & \text { if } \frac{2+n}{2} \leq \rho \leq n\end{cases}
$$

The distance-vector representations are distinct for all the vertices of $S\left(N_{e_{n}}\right)$. So, $W$ is the RS. Hence, $\beta\left(S\left(N_{e_{n}}\right)\right) \leq 3$. Now, in order to prove that $\beta\left(S\left(N_{e_{n}}\right)\right) \geq 3$, suppose contrary that $\beta\left(S\left(N_{e_{n}}\right)\right)=2$, and according to Lemma 3, we have the following possibilities:

(1) Let $W=\left\{x_{i}, x_{j}\right\} \subset V\left(S\left(N_{e_{n}}\right)\right)$ for $0 \leq i<j \leq$ $n+2 / 2$; then, $A D\left(\left(v_{n}, w_{n}\right) \mid W\right)=(0,0)$. So, $W$ is not the RS.

(2) Let $W=\left\{u_{i}, u_{j}\right\} \subset V\left(S\left(N_{e_{n}}\right)\right)$ for $1 \leq i<j \leq n+2 / 2$; then, $A D\left(\left(v_{n}, w_{n}\right) \mid W\right)=(0,0)$. So, $W$ is not the RS.

(3) Let $W=\left\{x_{i}, x_{j}\right\} \subset V\left(S\left(N_{e_{n}}\right)\right)$ for $0 \leq i \leq n / 2$ and $n+2 / 2 \leq j \leq n+1$; then, we have the following subcases:

(a) When $n=4$ :

(i) $A D\left(\left(v_{4}, w_{4}\right) \mid W\right)=(0,0)$ for $j \neq 4$.

(ii) $A D\left(\left(v_{1}, w_{2}\right) \mid W\right)=(0,0)$ for $i \neq 1$ and $j=4$.

(iii) $A D\left(\left(u_{1}, u_{2}\right) \mid W\right)=(0,0)$ for $i=1$ and $j=4$.

(b) When $n \geq 6$ :

(i) $A D\left(\left(v_{n}, w_{n}\right) \mid W\right)=(0,0)$ for $j \neq n$.

(ii) $A D\left(\left(u_{n}, v_{n}\right) \mid W\right)=(0,0)$ for $0 \leq i \leq n / 2-2$ and $j=n$.

(iii) $A D\left(\left(v_{1}, w_{2}\right) \mid W\right)=(0,0)$ for $n / 2-1 \leq i \leq$ $n / 2$ and $j=n$.

So, $W$ is not the RS.

(4) Let $\quad W=\left\{u_{i}, u_{j}\right\} \subset V\left(S\left(N_{e_{n}}\right)\right) \quad$ for $\quad 1 \leq i \leq$ $n / 2<j \leq n+1$; then, we have the following subcases:

(a) When $n=4$ :

(i) $A D\left(\left(v_{4}, w_{4}\right) \mid W\right)=(0,0)$ for $j=3$.

(ii) $A D\left(\left(v_{1}, w_{1}\right) \mid W\right)=(0,0)$ for $i=1$ and $j=4$.

(iii) $A D\left(\left(w_{1}, w_{2}\right) \mid W\right)=(0,0)$ for $i=2$ and $j=4$.

(iv) $A D\left(\left(u_{4}, v_{4}\right) \mid W\right)=(0,0)$ for $i=1$ and $j=5$.

(v) $A D\left(\left(x_{1}, x_{2}\right) \mid W\right)=(0,0)$ for $i=2$ and $j=5$.

(b) When $n=6$ :

(i) $A D\left(\left(v_{6}, w_{6}\right) \mid W\right)=(0,0)$ for $n / 2+1 \leq j \leq$ $n-1$.

(ii) $A D\left(\left(v_{2}, w_{2}\right) \mid W\right)=(0,0)$ for $i=1$ and $j=6$. (iii) $A D\left(\left(x_{1}, x_{2}\right) \mid W\right)=(0,0)$ for $i=2$ and $j=6$.

(iv) $A D\left(\left(v_{1}, w_{2}\right) \mid W\right)=(0,0)$ for $i=3$ and $j=6$.

(v) $A D\left(\left(u_{6}, v_{6}\right) \mid W\right)=(0,0)$ for $i \neq 3$ and $j=7$.

(vi) $A D\left(\left(x_{2}, x_{3}\right) \mid W\right)=(0,0)$ for $i=3$ and $j=7$.

(c) When $n \geq 8$ :

(i) $A D\left(\left(v_{n}, w_{n}\right) \mid W\right)=(0,0)$ for $n / 2+1 \leq j \leq$ $n-1$.

(ii) $A D\left(\left(v_{n / 2-1}, w_{n / 2-1}\right) \mid W\right)=(0,0)$ for $1 \leq i \leq$ $n / 2-2$ and $j=n$.

(iii) $A D\left(\left(v_{1}, w_{2}\right) \mid W\right)=(0,0)$ for $n / 2-1 \leq i \leq n / 2$ and $j=n$.

(iv) $A D\left(\left(u_{n}, v_{n}\right) \mid W\right)=(0,0)$ for $i \neq n / 2$ and $j=n+1$.

(v) $A D\left(\left(x_{n / 2-1}, x_{n / 2}\right) \mid W\right)=(0,0)$ for $i=n / 2$ and $j=n+1$.

So, $W$ is not the RS.

(5) Let $W=\left\{x_{i}, u_{j}\right\} \subset V\left(S\left(N_{e_{n}}\right)\right)$ for $0 \leq i \leq n / 2$ and $1 \leq j \leq n+2 / 2$; then, $A D\left(\left(v_{n}, w_{n}\right) \mid W\right)=(0,0)$. So, $W$ is not the RS.

(6) Let $W=\left\{x_{i}, v_{j}\right\} \subset V\left(S\left(N_{e_{n}}\right)\right)$ for $0 \leq i \leq n / 2$ and $1 \leq j \leq n / 2$; then, we have the following subcases:

(i) $A D\left(\left(v_{n+1}, w_{n+1}\right) \mid W\right)=(0,0)$ for $i \neq n / 2$.

(ii) $A D\left(\left(u_{n / 2+1}, v_{n / 2}\right) \mid W\right)=(0,0)$ for $i=n / 2$ and $j \neq n / 2$.

(iii) $A D\left(\left(u_{n / 2}, u_{n / 2+1}\right) \mid W\right)=(0,0)$ for $i=n / 2$ and $j=n / 2$.

So, $W$ is not the RS.

(7) Let $W=\left\{u_{i}, v_{j}\right\} \subset V\left(S\left(N_{e_{n}}\right)\right)$ for $1 \leq i \leq n+2 / 2$ and $1 \leq j \leq n / 2$; then, we have the following subcases:

(i) $A D\left(\left(u_{n+1}, w_{n+1}\right) \mid W\right)=(0,0)$ for $i \neq n+2 / 2$.

(ii) $A D\left(\left(u_{n / 2+2}, v_{n / 2+1}\right) \mid W\right)=(0,0)$ for $i=n+2 / 2$. So, $W$ is not the RS.

(8) Let $W=\left\{x_{i}, y_{j}\right\} \subset V\left(S\left(N_{e_{n}}\right)\right)$ for $0 \leq i \leq n / 2$ and $1 \leq j \leq n / 2$; then, we have the following subcases:

(i) $A D\left(\left(u_{n+1}, w_{n+1}\right) \mid W\right)=(0,0)$ for $i \neq n / 2$ and $j \neq n / 2$.

(ii) $A D\left(\left(u_{n / 2}, v_{n / 2}\right) \mid W\right)=(0,0)$ for $i=n / 2$ and $j \neq n / 2$.

(iii) $A D\left(\left(u_{n}, v_{n}\right) \mid W\right)=(0,0)$ for $i=0$ and $j=n / 2$. 
(iv) $A D\left(\left(v_{n / 2}, w_{n / 2}\right) \mid W\right)=(0,0)$ for $1 \leq i \leq n-2 / 2$ and $j=n / 2$.

(v) $A D\left(\left(u_{n / 2}, u_{n / 2+1}\right) \mid W\right)=(0,0)$ for $i=n / 2$ and $j=n / 2$.

So, $W$ is not the RS.

(9) Let $W=\left\{u_{i}, w_{j}\right\} \subset V\left(S\left(N_{e_{n}}\right)\right)$ for $1 \leq i \leq n+2 / 2$ and $1 \leq j \leq n+2 / 2$; then, we have the following subcases:

(i) $A D\left(\left(u_{n+1}, w_{n+1}\right) \mid W\right)=(0,0)$ for $i \neq n+2 / 2$ and $j \neq n+2 / 2$.

(ii) $A D\left(\left(v_{n}, w_{n}\right) \mid W\right)=(0,0)$ for $i=n+2 / 2$ and $1 \leq j \leq n-2 / 2$.

(iii) $A D\left(\left(x_{n-2 / 2}, y_{n+2 / 2}\right) \mid W\right)=(0,0)$ for $i=n+2 / 2$ and $j=n / 2$.

(iv) $A D\left(\left(u_{n}, v_{n}\right) \mid W\right)=(0,0)$ for $1 \leq i \leq n-2 / 2$ and $j=n+2 / 2$.

(v) $A D\left(\left(x_{n+2 / 2}, y_{n-2 / 2}\right) \mid W\right)=(0,0)$ for $i=n / 2$ and $j=n+2 / 2$.

(vi) $A D\left(\left(x_{n / 2}, x_{n / 2+1}\right) \mid W\right)=(0,0)$ for $i=n+2 / 2$ and $j=n+2 / 2$.

So, $W$ is not the RS.

(10) Let $W=\left\{u_{i}, y_{j}\right\} \subset V\left(S\left(N_{e_{n}}\right)\right)$ for $1 \leq i \leq n+2 / 2$ and $1 \leq j \leq n / 2$; then, we have the following subcases:

(i) $A D\left(\left(u_{n+1}, w_{n+1}\right) \mid W\right)=(0,0)$ for $i \neq n+2 / 2$ and $j \neq n / 2$. (ii) $A D\left(\left(u_{n / 2}, v_{n / 2}\right) \mid W\right)=(0,0)$ for $i=n+2 / 2$ and $j \neq n / 2$.

(iii) $A D\left(\left(v_{n / 2}, w_{n / 2}\right) \mid W\right)=(0,0)$ for $1 \leq i \leq n-2 / 2$ and $j=n / 2$.

(iv) $A D\left(\left(x_{n / 2}, x_{n / 2+1}\right) \mid W\right)=(0,0)$ for $n / 2 \leq i \leq n+$ $2 / 2$ and $j=n / 2$.

So, $W$ is not the RS.

We deduce to contradict in all the possibilities. So, there is no RS with cardinality 2 . This shows that $\beta\left(S\left(N_{e_{n}}\right)\right) \geq 3$. Hence, $\beta\left(S\left(N_{e_{n}}\right)\right)=3$.

Now, the following theorem presented the exact value of the FTMD of the subdivision graph of the necklace graph.

Theorem 3. For any integer $n \geq 3$, we have $\beta^{\prime}\left(S\left(N_{e_{n}}\right)\right)=4$.

Proof. Following are the cases to calculate required results:

Case 3. (when $n$ is odd).

Take $W^{\prime}=\left\{x_{0}, u_{n+1 / 2}, v_{n+1 / 2}, w_{n+1 / 2}\right\} \subseteq V\left(S\left(N_{e_{n}}\right)\right)$ for all odd integers $n \geq 3$. We need to prove that $W^{\prime}$ is the FTRS for the graph $S\left(N_{e_{n}}\right)$. To prove this, we give distance-vector representations of every vertex in $S\left(N_{e_{n}}\right)$. Representation of the vertices $x_{\rho}$ where $0 \leq \rho \leq 1+n$ is

$$
r\left(x_{\rho} \mid W^{\prime}\right)= \begin{cases}(0, n, n+2, n), & \text { if } \rho=0, \\ (2 \rho,-2 \rho+n, 2(1-\rho)+n, 2(1-\rho)+n), & \text { if } 1 \leq \rho \leq \frac{n-1}{2}, \\ (1+n, 1,1,3), & \text { if } \rho=\frac{1+n}{2}, \\ (-2 \rho+4+2 n, 2 \rho-n,-n+2 \rho,-n+2(1+\rho)), & \text { if } \frac{3+n}{2} \leq \rho \leq n, \\ (2, n+2,2+n, n+2), & \text { if } \rho=1+n .\end{cases}
$$

Representation of the vertices $u_{\rho}$ where $1 \leq \rho \leq 1+n$ is

$$
r\left(u_{\rho} \mid W^{\prime}\right)= \begin{cases}(-1+2 \rho,-2 \rho+1+n,-2 \rho+3+n,-2 \rho+3+n), & \text { if } 1 \leq \rho \leq \frac{n-1}{2}, \\ (n, 0,2,4), & \text { if } \rho=\frac{1+n}{2}, \\ (5-2(\rho-n),-n-1+2 \rho,-1+2 \rho-n,-n+1+2 \rho), & \text { if } \frac{3+n}{2} \leq \rho \leq 1+n .\end{cases}
$$


Representation of the vertices $w_{\rho}$ where $1 \leq \rho \leq 1+n$ is

$$
r\left(w_{\rho} \mid W^{\prime}\right)= \begin{cases}(-1+2 \rho,-2 \rho+3+n,-2 \rho+3+n,-2 \rho+n+1), & \text { if } 1 \leq \rho \leq \frac{n-1}{2}, \\ (n, 4,2,0), & \text { if } \rho=\frac{1+n}{2}, \\ (-2 \rho+5+2 n,-n+1+2 \rho,-n-1+2 \rho,-1-n+2 \rho), & \text { if } \frac{3+n}{2} \leq \rho \leq 1+n .\end{cases}
$$

Representation of the vertices $v_{\rho}$ where $1 \leq \rho \leq 1+n$ is

$$
r\left(v_{\rho} \mid W^{\prime}\right)= \begin{cases}(2 \rho+1,-2 \rho+1+n,-2 \rho+3+n,-2 \rho+n+1), & \text { if } 1 \leq \rho \leq \frac{n-1}{2}, \\ (2+n, 2,0,2), & \text { if } \rho=\frac{1+n}{2}, \\ (5-2 \rho+2 n,-n+1+2 \rho,-n+1+2 \rho,-n+2 \rho+1), & \text { if } \frac{3+n}{2} \leq \rho \leq n, \\ (1, n+1,3+n, 1+n), & \text { if } \rho=1+n .\end{cases}
$$

Representation of the vertices $y_{\rho}$ where $1 \leq \rho \leq n$ is

$$
r\left(y_{\rho} \mid W^{\prime}\right)= \begin{cases}(2 \rho,-2 \rho+2+n,-2 \rho+2+n,-2 \rho+n), & \text { if } 1 \leq \rho \leq \frac{n-1}{2}, \\ (1+n, 3,1,1), & \text { if } \rho=\frac{1+n}{2}, \\ (-2 \rho+4+2 n,-n+2+2 \rho, 2 \rho-n,-n+2 \rho), & \text { if } \frac{3+n}{2} \leq \rho \leq n .\end{cases}
$$

The distance-vector representations are distinct for all the vertices of $S\left(N_{e_{n}}\right)$. So, $W^{\prime}$ is the RS. We can also verify that, for every $x, y \in V\left(S\left(N_{e_{n}}\right)\right)$, at least two elements in the 4-vector $A D\left((x, y) \mid W^{\prime}\right)$ are nonzero. So, the RS $W^{\prime}$ becomes a FTRS. This shows that $\beta^{\prime}\left(S\left(N_{e_{n}}\right)\right) \leq 4$. By using
Lemma 1 and Theorem 2, we have $\beta^{\prime}\left(S\left(N_{e_{n}}\right)\right) \geq 4$. Hence, $\beta^{\prime}\left(S\left(N_{e_{n}}\right)\right)=4$.

Case 4. (when $n$ is even). 
Take $W^{\prime}=\left\{x_{0}, x_{n / 2}, v_{n+4 / 2}, y_{n / 2}\right\} \subseteq V\left(S\left(N_{e_{n}}\right)\right)$ for all even integers $n \geq 4$. Representation of the vertices $x_{\rho}$ where $0 \leq \rho \leq 1+n$ is

$$
r\left(x_{\rho} \mid W^{\prime}\right)= \begin{cases}(0, n, 1+n, n), & \text { if } \rho=0, \\ (2 \rho,-2 \rho+n,-2 \rho+5+n,-2 \rho+2+n), & \text { if } 1 \leq \rho \leq \frac{n}{2}, \\ (2+n, 2,3,4), & \text { if } \rho=\frac{2+n}{2}, \\ (-2 \rho+4+2 n,-n+2 \rho,-n-3+2 \rho,-n+2+2 \rho), & \text { if } \frac{4+n}{2} \leq \rho \leq n \\ (2, n+2,-1+n, 2+n), & \text { if } \rho=1+n .\end{cases}
$$

Representation of the vertices $u_{\rho}$ where $1 \leq \rho \leq 1+n$ is

$$
r\left(u_{\rho} \mid W^{\prime}\right)= \begin{cases}(1,-1+n, n+2,1+n), & \text { if } \rho=1, \\ (-1+2 \rho,-2 \rho+1+n,-2 \rho+6+n,-2 \rho+3+n), & \text { if } 2 \leq \rho \leq \frac{n}{2}, \\ (1+n, 1,4,3), & \text { if } \rho=\frac{2+n}{2}, \\ (1+n, 3,2,5), & \text { if } \rho=\frac{4+n}{2}, \\ (5-2 \rho+2 n,-n-1+2 \rho,-n-4+2 \rho,-n+1+2 \rho), & \text { if } \frac{6+n}{2} \leq \rho \leq 1+n .\end{cases}
$$

Representation of the vertices $w_{\rho}$ where $1 \leq \rho \leq 1+n$ is

$$
r\left(w_{\rho} \mid W^{\prime}\right)= \begin{cases}(1,1+n, 2+n,-1+n), & \text { if } \rho=1, \\ (-1+2 \rho,-2 \rho+3+n,-2(\rho-3)+n,-2 \rho+n+1), & \text { if } 2 \leq \rho \leq \frac{n}{2}, \\ (1+n, 3,4,1), & \text { if } \rho=\frac{2+n}{2}, \\ (1+n, 5,2,3), & \text { if } \rho=\frac{4+n}{2}, \\ (-2 \rho+5+2 n,-n+1+2 \rho,-4-n+2 \rho,-1-n+2 \rho), & \text { if } \frac{6+n}{2} \leq \rho \leq 1+n .\end{cases}
$$


Representation of the vertices $v_{\rho}$ for $n=4$ where $1 \leq \rho \leq 5$ is
Representation of the vertices $v_{\rho}$ for $n>4$ where $1 \leq \rho \leq 1+n$ is

$$
r\left(v_{\rho} \mid W^{\prime}\right)= \begin{cases}(1+2 \rho, 1-2 \rho+n,-4-2 \rho+2 n, 1-2 \rho+n), & \text { if } 1 \leq \rho \leq \frac{n}{2}, \\ (3+n, 3,4,3), & \text { if } \rho=\frac{2+n}{2}, \\ (1+n, 5,0,5), & \text { if } \rho=\frac{4+n}{2}, \\ (5-2 \rho+2 n,-n+1+2 \rho,-n-2+2 \rho,-n+1+2 \rho), & \text { if } \frac{6+n}{2} \leq \rho \leq n, \\ (1, n+1, n, 1+n), & \text { if } \rho=1+n .\end{cases}
$$

Representation of the vertices $y_{\rho}$ where $1 \leq \rho \leq n$ is

$$
r\left(y_{\rho} \mid W^{\prime}\right)= \begin{cases}(2 \rho,-2 \rho+2+n,-2 \rho+5+n,-2 \rho+n), & \text { if } 1 \leq \rho \leq \frac{n}{2}, \\ (2+n, 4,3,2), & \text { if } \rho=\frac{2+n}{2}, \\ (-2 \rho+4+2 n,-n+2(1+\rho),-n-3+2 \rho,-n+2 \rho), & \text { if } \frac{4+n}{2} \leq \rho \leq n .\end{cases}
$$

The distance-vector representations are distinct for all the vertices of $S\left(N_{e_{n}}\right)$. So, $W^{\prime}$ is the RS. We can also verify that, for every $x, y \in V\left(S\left(N_{e_{n}}\right)\right)$, at least two elements in the 4 -vector $A D\left((x, y) \mid W^{\prime}\right)$ are nonzero. So, the RS $W^{\prime}$ becomes a FTRS. This shows that $\beta^{\prime}\left(S\left(N_{e_{n}}\right)\right) \leq 4$. By using Lemma 1 and Theorem 2, we have $\beta^{\prime}\left(S\left(N_{e_{n}}\right)\right) \geq 4$. Hence, $\beta^{\prime}\left(S\left(N_{e_{n}}\right)\right)=4$.

\section{The Fault-Tolerant Metric Dimension for the Subdivision of the Prism Graph}

The prism graph $Y_{n}$ is Cartesian product graph $C_{n} \times P_{2}$, where $C_{n}$ is the cycle graph of order $n$ and $P_{2}$ is a path of order 2. The prism graph $Y_{n}$ consists of 4-sided faces and $n$-sided faces with vertex set $V\left(Y_{n}\right)=\left\{x_{k}, y_{k} ; 1 \leq k \leq n\right\}$ as shown in Figure 4.
Now, to compute $\beta^{\prime}\left(S\left(Y_{n}\right)\right)$, we need to convert the prism graph into its subdivision graph. The subdivision graph of the prism graph $S\left(Y_{n}\right)$ is shown in Figure 2 .

The known result of the MD of the subdivision graph of the prism graph is given in the following theorem.

Theorem 4 (see [26]). For any integer $n \geq 4$, we have $\beta\left(S\left(Y_{n}\right)\right)=3$.

Here, we will find the exact value of $\beta^{\prime}\left(S\left(Y_{n}\right)\right)$, which is presented in the following theorem.

Theorem 5. For any integer $n \geq 3$, we have $\beta^{\prime}\left(S\left(Y_{n}\right)\right)=4$.

Proof. Following are the cases to compute required results: 


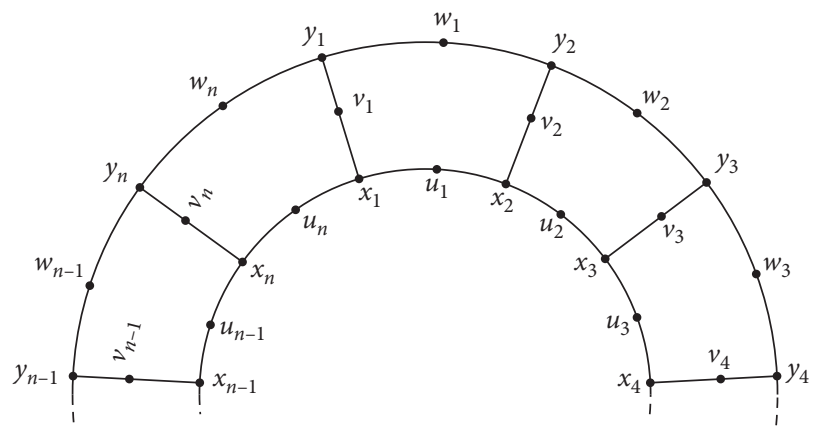

Figure 4: Prism graph. $Y_{n}$.

Case 5. (when $n$ is even).

Take $W^{\prime}=\left\{x_{1}, x_{2}, x_{n+2 / 2}, x_{n+4 / 2}\right\} \subseteq V\left(S\left(Y_{n}\right)\right)$ for all even integers $n \geq 4$. We need to prove that $W^{\prime}$ is the FTRS for the graph $S\left(Y_{n}\right)$. To prove this, we give distance-vector representations of every vertex in $S\left(Y_{n}\right)$. Representation of the vertices $x_{\rho}$ where $1 \leq \rho \leq n$ is

$$
r\left(x_{\rho} \mid W^{\prime}\right)= \begin{cases}(0,2, n,-2+n), & \text { if } \rho=1, \\ (-2(1-\rho),-2(2-\rho),-2 \rho+2+n,-2 \rho+4+n), & \text { if } 2 \leq \rho \leq \frac{2+n}{2} \\ (-2 \rho+2+2 n,-2 \rho+4+2 n,-2-n+2 \rho,-4-n+2 \rho), & \text { if } \frac{4+n}{2} \leq \rho \leq n .\end{cases}
$$

Representation of the vertices $u_{\rho}$ where $1 \leq \rho \leq n$ is

$$
r\left(u_{\rho} \mid W^{\prime}\right)= \begin{cases}(1,1,-1+n,-1+n), & \text { if } \rho=1, \\ (2 \rho-1,-3+2 \rho,-2 \rho+n+1,-2 \rho+3+n), & \text { if } 2 \leq \rho \leq \frac{n}{2}, \\ (-1+n,-1+n, 1,1), & \text { if } \rho=\frac{2+n}{2}, \\ (-2(\rho-n)+1,-2 \rho+3+2 n,-n-1+2 \rho,-n-3+2 \rho), & \text { if } \frac{4+n}{2} \leq \rho \leq n .\end{cases}
$$

Representation of the vertices $v_{\rho}$ where $1 \leq \rho \leq n$ is

$$
r\left(v_{\rho} \mid W^{\prime}\right)= \begin{cases}(1,3,1+n,-1+n), & \text { if } \rho=1, \\ (-1+2 \rho,-3+2 \rho,-2 \rho+3+n,-2 \rho+5+n), & \text { if } 2 \leq \rho \leq \frac{2+n}{2}, \\ (-2(\rho-n)+3,-2 \rho+5+2 n,-1-n+2 \rho,-n-3+2 \rho), & \text { if } \frac{4+n}{2} \leq \rho \leq n .\end{cases}
$$


Representation of the vertices $y_{\rho}$ where $1 \leq \rho \leq n$ is

$$
r\left(y_{\rho} \mid W^{\prime}\right)= \begin{cases}(2,4,2+n, n), & \text { if } \rho=1, \\ (2 \rho,-2(1-\rho),-2 \rho+4+n,-2 \rho+6+n), & \text { if } 2 \leq \rho \leq \frac{2+n}{2}, \\ (-2(\rho-2-n),-2(\rho-3-n),-n+2 \rho,-2-n+2 \rho), & \text { if } \frac{4+n}{2} \leq \rho \leq n .\end{cases}
$$

Representation of the vertices $w_{\rho}$ where $1 \leq \rho \leq n$ is

$$
r\left(w_{\rho} \mid W^{\prime}\right)= \begin{cases}(3,3,1+n, 1+n), & \text { if } \rho=1, \\ (1+2 \rho,-1+2 \rho,-2 \rho+3+n,-2 \rho+5+n), & \text { if } 2 \leq \rho \leq \frac{n}{2}, \\ (1+n, 1+n, 3,3), & \text { if } \rho=\frac{2+n}{2}, \\ (-2 \rho+3+2 n,-2 \rho+5+2 n,-n+1+2 \rho,-n-1+2 \rho), & \text { if } \frac{4+n}{2} \leq \rho \leq n .\end{cases}
$$

The distance-vector representations are distinct for all the vertices of $S\left(Y_{n}\right)$. So, $W^{\prime}$ is the RS. We can also verify that, for every $x, y \in V\left(S\left(Y_{n}\right)\right)$, at least two elements in the 4 -vector $A D\left((x, y) \mid W^{\prime}\right)$ are nonzero. So, the RS $W^{\prime}$ becomes a FTRS. This shows that $\beta^{\prime}\left(S\left(Y_{n}\right)\right) \leq 4$. By using Lemma 1 and Theorem 4 , we have $\beta^{\prime}\left(S\left(Y_{n}\right)\right) \geq 4[0,1]$. Hence, $\beta^{\prime}\left(S\left(Y_{n}\right)\right)=4$.
Case 6. (when $n$ is odd).

For $n=3$, take $W^{\prime}=\left\{x_{1}, x_{2}, y_{1}, y_{2}\right\} \subseteq V\left(L\left(Y_{3}\right)\right)$. It is quite simple to check that $W^{\prime}$ is a FTRS.

Take $W^{\prime}=\left\{x_{1}, x_{2}, y_{1}, y_{2}\right\} \subseteq V\left(L\left(Y_{n}\right)\right)$ for all odd integers $n \geq 5$. Representation of the vertices $x_{\rho}$ where $1 \leq \rho \leq n$ is

$$
r\left(x_{\rho} \mid W^{\prime}\right)= \begin{cases}(0,2,2,4), & \text { if } \rho=1, \\ (-2(1-\rho),-2(2-\rho), 2 \rho,-2(1-\rho)), & \text { if } 2 \leq \rho \leq \frac{1+n}{2}, \\ (-1+n, n-1,1+n, 1+n), & \text { if } \rho=\frac{3+n}{2}, \\ (-2(\rho-1-n),-2(\rho-2-n),-2 \rho+4+2 n,-2(\rho-3-n)), & \text { if } \frac{5+n}{2} \leq \rho \leq n .\end{cases}
$$

Representation of the vertices $u_{\rho}$ where $1 \leq \rho \leq n$ is 


$$
r\left(u_{\rho} \mid W^{\prime}\right)= \begin{cases}(1,1,3,3), & \text { if } \rho=1, \\ (2 \rho-1,-3+2 \rho, 2 \rho+1,-1+2 \rho), & \text { if } 2 \leq \rho \leq \frac{1+n}{2}, \\ (-2(\rho-n)+1,-2 \rho+3+2 n,-2 \rho+3+2 n,-2(\rho-n)+5), & \text { if } \frac{3+n}{2} \leq \rho \leq n .\end{cases}
$$

Representation of the vertices $v_{\rho}$ where $1 \leq \rho \leq n$ is

$$
r\left(v_{\rho} \mid W^{\prime}\right)= \begin{cases}(1,3,1,3), & \text { if } \rho=1, \\ (-1+2 \rho,-3+2 \rho, 2 \rho-1,-3+2 \rho), & \text { if } 2 \leq \rho \leq \frac{1+n}{2}, \\ (n, n, n, n), & \text { if } \rho=\frac{3+n}{2}, \\ (-2(\rho-n)+3,-2 \rho+5+2 n,-2 \rho+3+2 n,-2(\rho-n)+5), & \text { if } \frac{5+n}{2} \leq \rho \leq n .\end{cases}
$$

Representation of the vertices $y_{\rho}$ where $1 \leq \rho \leq n$ is

$$
r\left(y_{\rho} \mid W^{\prime}\right)= \begin{cases}(2,4,0,2), & \text { if } \rho=1, \\ (2 \rho,-2(1-\rho),-2+2 \rho,-2(2-\rho)), & \text { if } 2 \leq \rho \leq \frac{1+n}{2}, \\ (1+n, n+1, n-1,-1+n), & \text { if } \rho=\frac{3+n}{2}, \\ (-2 \rho+4+2 n,-2(\rho-3-n),-2 \rho+2+2 n,-2 \rho+4+2 n), & \text { if } \frac{5+n}{2} \leq \rho \leq n .\end{cases}
$$

Representation of the vertices $w_{\rho}$ where $1 \leq \rho \leq n$ is

$$
r\left(w_{\rho} \mid W^{\prime}\right)= \begin{cases}(3,3,1,1), & \text { if } \rho=1, \\ (1+2 \rho,-1+2 \rho, 2 \rho-1,-3+2 \rho), & \text { if } 2 \leq \rho \leq \frac{1+n}{2} \\ (-2(\rho-n)+3,-2 \rho+5+2 n,-2 \rho+1+2 n,-2(\rho-n)+3), & \text { if } \frac{3+n}{2} \leq \rho \leq n\end{cases}
$$


The distance-vector representations are distinct for all the vertices of $S\left(Y_{n}\right)$. So, $W^{\prime}$ is the RS. We can also verify that, for every $x, y \in V\left(S\left(Y_{n}\right)\right)$, at least two elements in the 4 -vector $A D\left((x, y) \mid W^{\prime}\right)$ are nonzero. So, the RS $W^{\prime}$ becomes a FTRS. This shows that $\beta^{\prime}\left(S\left(Y_{n}\right)\right) \leq 4$. By using Lemma 1 and Theorem 4, we have $\beta^{\prime}\left(S\left(Y_{n}\right)\right) \geq 4$. Hence, $\beta^{\prime}\left(S\left(Y_{n}\right)\right)=4$.

\section{Conclusion}

In this paper, we have studied, for the first time, the FTMD of the subdivision graph of a graph. We have found that the FTMD of the subdivision graphs of the necklace and prism graphs is exactly greater than one of their metric dimensions.

\section{Data Availability}

No data were used in this manuscript.

\section{Conflicts of Interest}

The authors declare that they have no conflicts of interest.

\section{References}

[1] F. Deng, X. Zhang, M. Alaeiyan, A. Mehboob, and M. R. Farahani, "Topological indices of the pent-heptagonal nanosheets VC5C7 and $\mathrm{HC5C7,"} \mathrm{Advances} \mathrm{in} \mathrm{Materials}$ Science and Engineering, 2019.

[2] A. Farooq, M. Habib, A. Mahboob, W. Nazeer, and S. M. Kang, "Zagreb polynomials and redefined zagreb indices of dendrimers and polyomino chains," Open Chemistry, vol. 17, no. 1, pp. 1374-1381, 2019.

[3] P. J. Slater, "Leaves of trees," Congressus Numerantium, vol. 14, pp. 549-559, 1975.

[4] P. J. Slater, "Dominating and reference set in a graph," Journal of Mathematical and Physical Sciences, vol. 22, no. 4, pp. 445-455, 1988.

[5] F. Harary and R. A. Melter, "On the metric dimension of a graph,” Ars Combinatoria, vol. 2, pp. 191-195, 1976.

[6] G. Chartrand, L. Eroh, M. A. Johnson, and O. R. Oellermann, "Resolvability in graphs and the metric dimension of a graph," Discrete Applied Mathematics, vol. 2019, Article ID 9594549, 2000.

[7] G. Chartrand and P. Zhang, "The theory and applications of resolvability in graphs," A survey. Congressus Numerantium.vol. 160, pp. 47-68, 2003.

[8] Y. Tan, J. Zhang, H. Tian et al., "Multi-label classification for simultaneous fault diagnosis of marine machinery: a comparative study," Ocean Engineering, vol. 239, Article ID 109723, 2021.

[9] C. Hernando, M. Mora, P. J. Slater, and D. R. Wood, "Faulttolerant metric dimension of graph," Proceedings of the International Conference Convexity in Discrete Structure, Ramanujan Math. Society Lecture Notes, vol. 5, pp. 81-85, 2008.

[10] R. V. Voronov, "The fault-tolerant metric dimension of the king's graph," Vestnik of Saint Petersburg University. Applied Mathematics. Computer Science. Control Processes, vol. 13, no. 3, pp. 241-249, 2017.

[11] Z. Hussain, M. Munir, I. Ali, A. Ahmad, and S. Kang, "Fault-tolerant metric dimension of wheel related graphs," Mathmatical Problems in Engineering, hal-01857316v2, 2019.
[12] H. Raza, S. Hayat, and X.-F. Pan, "On the fault-tolerant metric dimension of convex polytopes," Applied Mathematics and Computation, vol. 339, pp. 172-185, 2018.

[13] H. Raza, S. Hayat, M. Imran, and X.-F. Pan, "Fault-tolerant resolvability and extremal structures of graphs," Mathematics, vol. 7, no. 1, p. 78, 2019.

[14] J. B. Liu, M. Munir, I. Ali, Z. Hussain, and A. Ahmed, "Faulttolerant metric dimension of wheelrelated graphs," Journal of Applied Mathematics and Computing, 2019, https://hal. archives-ouvertes.fr/hal-01857316v2.

[15] Z. B. Zheng, A. Ahmad, Z. Hussain et al., "Fault-tolerant metric dimension of generalized wheels and convex polytopes," Mathematical Problems in Engineering, vol. 2020, p. 8, Article ID 1216542, 2020.

[16] H. M. A. Siddiqui, S. Hayat, A. Khan, M. Imran, A. Razzaq, and J.-B. Liu, "Resolvability and fault-tolerant resolvability structures of convex polytopes," Theoretical Computer Science, vol. 796, pp. 114-128, 2019.

[17] M. Basak, L. Saha, G. K. Das, and K. Tiwary, "Fault-tolerant metric dimension of circulant graphs $\mathrm{C}(1,2,3)$," Theoretical Computer Science, vol. 817, pp. 66-79, 2020.

[18] M. Somasundari and F. S. Raj, "Fault-tolerant resolvability of oxide interconnections," International Journal of Innovative Technology and Exploring Engineering, vol. 8, no. 12, pp. 2278-3075, 2019.

[19] I. Javaid, M. Salman, M. A. Chaudhry, and S. Shokat, "Faulttolerance in resolvability," Utilitas Mathematica, vol. 80, pp. 263-275, 2009.

[20] S. Hayat, A. Khan, M. Y. H. Malik, M. Imran, and M. K. Siddiqui, "Fault-tolerant metric dimension of interconnection networks," IEEE Access, vol. 8, pp. 145435-145445, 2020.

[21] A. Simic, M. Bogdanovic, Z. Maksimovic, and J. Milosevic, "faulttolerant metric dimension problem: a new integer linear programming formulation and exact formula for grid graph," Krag. Journal of Math, vol. 42, no. 4, pp. 495-503, 2018.

[22] L. Saha, "Fault-tolerant metric dimension of cube of paths," IOP Publishing, vol. 1714, no. No. 1, Journal of Physics: Conference Series, Article ID 012029, 2021.

[23] X. Guo, M. Faheem, Z. Zahid, W. Nazeer, and J. Li, "Faulttolerant resolvability in some classes of line graphs," Mathmatical Problems in Engineering, vol. 2020, Article ID 1436872, 8 pages, 2020.

[24] A. Estrado-Moreno, J. A. Rodriguez-Velaquez, and I. G. Yero, "The $k$ - metric dimension of a graph," Applied Mathematics and Information Sciences, vol. 9, no. 6, pp. 2829-2840, 2015.

[25] S. Khuller, B. Raghavachari, and A. Rosenfeld, "Landmarks in graphs," Discrete Applied Mathematics, vol. 70, no. 3, pp. 217-229, 1996.

[26] M. Imran, "On the metric dimension of barycentric subdivision of cayley graphs," Acta Mathematicae Applicatae Sinica, English Series, vol. 32, no. 4, pp. 1067-1072, 2016. 